\title{
Impacto del fenómeno de migración de estadio ganglionar en la supervivencia y la recurrencia de pacientes con cáncer papilar de tiroides sometidos a vaciamiento ganglionar profiláctico
}

\author{
Impact of the node-stage migration phenomenon on survival and \\ recurrence of patients with papillary thyroid cancer who underwent \\ prophylactic lymph node dissection
}

José Eduardo Agamez-Fuentes ${ }^{1} \mathbb{D}$, Álvaro Enrique Sanabria² $\mathbb{D}$

1 Médico, residente de Cirugía general, Universidad de Antioquia, Medellín, Colombia.

2 Médico, especialista en Cirugía general, magister en Epidemiología Clínica y Economía de la Salud, doctorado en Oncología de Cabeza y Cuello, CEXCA (Centro de Excelencia en Enfermedades de Cabeza y Cuello); profesor, Departamento de Cirugía, Facultad de Medicina, Universidad de Antioquia, Medellín, Colombia.

\begin{abstract}
Resumen
Introducción. Dado que un ensayo clínico aleatorio es irrealizable, el rol del vaciamiento ganglionar profiláctico en pacientes con cáncer papilar de tiroides sin comprobación clínica de compromiso ganglionar metastásico (cN0) es controversial. El vaciamiento ganglionar profiláctico acarrea un proceso de reclasificación de pacientes, al hacer evidente la positividad ganglionar micrometastásica antes ignorada, lo que genera una aparente pero falsa mejoría en los desenlaces de los grupos de estadificación, mientras el pronóstico individual y total de la población no cambia, fenómeno conocido como migración de estadio o fenómeno de Will Rogers.
\end{abstract}

Métodos. Se ejecutaron simulaciones de poblaciones con cáncer papilar de tiroides con compromiso ganglionar metastásico clínicamente evidente $(\mathrm{cN}+)$ y $\mathrm{cN} 0$, para determinar el impacto del fenómeno de migración de estadio en los pacientes sometidos a vaciamiento ganglionar profiláctico.

Resultados. Con la simulación de las poblaciones y sus estadios ganglionares, se observa cómo la migración de estadio ganglionar genera una aparente mejoría en los desenlaces de recurrencia loco regional y supervivencia, sin cambiar los desenlaces de la población total ni individuales.

Discusión. El fenómeno de migración de estadio es uno de los sesgos más importantes que limitan el uso de grupos históricos de control en ensayos de tratamiento experimental. De acuerdo con nuestros resultados, este fenómeno

Fecha de recibido: 15/09/2020 - Fecha de aceptación: 15/11/2020 - Fecha de publicación en línea: 10/06/2021

Correspondencia: José Eduardo Agamez-Fuentes, Calle 36 A \# 58 - 55, Bello, Colombia. Teléfono (+57) 3188664905.

Correo electrónico: joseeduardoagamez@gmail.com

Citar como: Agamez-Fuentes JE, Sanabria AE. Impacto del fenómeno de migración de estadio ganglionar en la supervivencia y la recurrencia de pacientes con cáncer papilar de tiroides sometidos a vaciamiento ganglionar profiláctico.

Rev Colomb Cir. 2021;36:599-610. https://doi.org/10.30944/20117582.853

Este es un artículo de acceso abierto bajo una Licencia Creative Commons - BY-NC-ND https://creativecommons.org/licenses/by-ncnd/4.0/deed.es 
podría explicar los beneficios observados con el vaciamiento ganglionar profiláctico en algunos de los estudios agregativos publicados hasta el momento, hallazgos que no han sido documentados para el cáncer papilar de tiroides.

Palabras clave: cáncer papilar tiroideo; escisión del ganglio linfático; migración de estadio ganglionar; disección del cuello central; recurrencia local de neoplasia; supervivencia.

\begin{abstract}
Introduction. The role of prophylactic central lymph node dissection at the time of total thyroidectomy remains controversial in clinically node-negative ( $\mathrm{cN} 0$ ) papillary thyroid carcinoma. Moreover, a prospective randomized controlled trial of prophylactic central lymph node dissection in cN0 RCT is not readily feasible.
\end{abstract}

Methods. In this study we simulated $\mathrm{cN} 0$ and clinically node-positive ( $\mathrm{cN}+$ ) populations, to evaluate impact of nodal stage migration in papillary thyroid carcinoma patients that undergo prophylactic central neck dissection. We use simulations of population and nodal stages.

Results. Nodal stage migration phenomenon seems to have an improvement in locoregional recurrence and overall survival of $\mathrm{cN} 0$ and $\mathrm{cN}+$ populations, without changes in overall population and individual outcomes.

Discussion. Nodal stage migration is recognized as an important bias that precludes the use of historical controls groups in experimental treatment trials. In accordance to our findings, this phenomenon could explain the improvements observed in outcomes in patients that undergo prophylactic central neck dissection.

Keywords: papillary thyroid cancer; lymph node excision; nodal stage migration; central neck dissection; local neoplasm recurrence; survival.

\section{Introducción}

El cáncer de tiroides es la neoplasia endocrina más común. Entre el 85 y el $95 \%$ de los pacientes con neoplasias tiroideas tienen un cáncer papilar de tiroides (CPT), el cual tiene una alta supervivencia a largo plazo. La cirugía, el yodo radioactivo y la supresión de hormona tiroidea son los pilares de manejo actual del $\mathrm{CPT}^{1-3}$.

Debido a la carencia de estudios prospectivos controlados, muchas de las recomendaciones de las guías de manejo acerca de la extensión de la cirugía, el papel del yodo radioactivo en el tratamiento de pacientes de bajo riesgo, la temporalidad y la frecuencia de la vigilancia, son controvertidas ${ }^{1}$.

En lo concerniente a la cirugía, existe consenso de que el vaciamiento ganglionar debe ser realizado ante la existencia de compromiso linfático palpable o detectado por imágenes, lo que se ha denominado vaciamiento terapéutico, y puede incluir el compartimiento central o lateral del cuello.
Sin embargo, en los pacientes con estadio clínico N0 (cN0), no existe claridad acerca del papel del vaciamiento ganglionar central profiláctico.

Se ha demostrado que las metástasis linfáticas regionales, en pacientes con cáncer de tiroides, impactan la recurrencia loco regional (RLR) y la mortalidad. No obstante, en casos cN0 no es claro el efecto de las micrometástasis existentes sobre los desenlaces de dichos pacientes. Además, la realización rutinaria del vaciamiento ganglionar puede llevar a un incremento de las tasas de complicaciones, incluyendo el hipoparatiroidismo permanente y la lesión del nervio laríngeo recurrente ${ }^{1,2}$.

Es claro que la supervivencia es mucho peor en pacientes con enfermedad metastásica regional que en los pacientes con enfermedad localizada. Debido a la precisión de los nuevos métodos, los pacientes con micrometástasis, que anteriormente se clasificaban en el grupo de tumores localizados, ahora son clasificados como pacientes con 
enfermedad metastásica regional, al hacer evidente la carga tumoral micrometastásica que antes era indetectable. Estos pacientes con pequeña carga tumoral metastásica tienen mejores tasas de supervivencia que los pacientes con procesos metastásicos masivos, pero peores tasas de supervivencia que los pacientes con cáncer no metastásico. Por tal razón, su transferencia del grupo de cáncer localizado al grupo de cáncer metastásico puede incrementar la supervivencia en ambos grupos, sin que cambie la supervivencia de cada individuo en particular, ni de la población total con cáncer. A esta paradoja epidemiológica se le conoce como fenómeno de migración de estadio o fenómeno de Will Rogers ${ }^{4-6}$.

Nuestra hipótesis es que el fenómeno de Will Rogers se encuentra mediando los aumentos aparentes en la supervivencia y la disminución en las tasas de recurrencia de los pacientes llevados a vaciamiento ganglionar central profiláctico en cáncer de tiroides $\mathrm{cN} 0$, como ya se ha demostrado en otros tumores, como cáncer de pulmón, estómago y colorrectal ${ }^{7-12}$.

Por tanto, nuestra pregunta de investigación planteada es: ¿Al comparar los pacientes adultos con cáncer papilar de tiroides sometidos a tiroidectomía total y vaciamiento ganglionar central profiláctico con los pacientes sometidos a tiroidectomía total, el fenómeno de migración de estadio explica el aumento en la supervivencia de los subgrupos de estadificación?

Para encontrar la solución a tal pregunta sería necesario un ensayo clínico aleatorizado para comparar la realización del vaciamiento ganglionar central profiláctico o no, en pacientes con CPT en estadio cN0. Sin embargo, el tamaño de muestra seria cercano a 5840 pacientes, requerirá un tiempo de seguimiento demasiado largo y unos gastos inadmisibles por un sistema de salud, por lo que dicho ensayo se ha considerado inviable ${ }^{1}$.

Otra alternativa para la resolución de dicha pregunta clínica es el diseño de un modelo matemático con parámetros poblacionales, en donde sea posible realizar simulación de la distribución poblacional de estadios ganglionares tumorales, para evaluar de manera sistemática el efecto de la migración de estadio ganglionar en la supervivencia de pacientes con cáncer papilar de tiroides que son llevados a vaciamiento ganglionar central profiláctico y comparar las tasas de supervivencia con el grupo de pacientes a quienes no se realizó tal procedimiento.

\section{Métodos}

Diseño: Este es un estudio secundario de modelación matemática basado en parámetros extraídos de la literatura.

Búsqueda de las fuentes primarias: La población de estudio fueron pacientes adultos con cáncer papilar de tiroides bien diferenciado, sin evidencia de compromiso metastásico ganglionar, clínico o por imágenes (cN0).

En la base de datos MEDLINE se realizó una búsqueda de los estudios publicados en los últimos 20 años respecto al vaciamiento ganglionar central profiláctico en cáncer papilar de tiroides. Se incluyeron artículos publicados en inglés donde se evaluaba el vaciamiento ganglionar central profiláctico en cáncer papilar de tiroides, la tasa de RLR y la supervivencia en los distintos grupos de estadificación. Se excluyeron los artículos que no mencionaban los desenlaces propuestos (RLR y la supervivencia en los distintos grupos de estadificación). En el anexo 1, puede encontrarse la estrategia de búsqueda usada en Pubmed para la revisión de los artículos potencialmente relevantes.

Generación de población cNO y de la distribución de pacientes con micrometástasis: A partir de los datos obtenidos se procedió a generar una población hipotética de pacientes $\mathrm{cN} 0$, que poseyeran la distribución de los factores de riesgo identificados en la literatura (sexo, edad, multifocalidad, tamaño del tumor, localización, invasión linfovascular, extensión extratiroidea, invasión capsular) ${ }^{4,5}$.

Los datos extraídos para el modelo se pueden observar en la tabla 1. Para adjudicar estos factores de riesgo, se utilizaron distribuciones de probabilidad binomial con los parámetros defi- 
Tabla 1. Parámetros extraídos para la generación del modelo.

\begin{tabular}{|c|c|c|c|}
\hline Parámetro & Valor & Fuente & Observaciones \\
\hline $\begin{array}{l}\text { Sexo: } \\
\text { a: Masculino } \\
\text { b: Femenino } \\
\text { rda: compromiso nodal para a } \\
\text { rdb: compromiso nodal para b }\end{array}$ & $\begin{array}{c}\mathrm{pa}=0,149 \\
\mathrm{rda}=0,5892 \\
\mathrm{rdb}=0,4711\end{array}$ & Sun et al., $2015^{5}$ & $\begin{array}{c}14,9 \% \text { masculinos, con el } \\
58,9 \% \text { de micrometástasis. }\end{array}$ \\
\hline $\begin{array}{l}\text { Edad: } \\
\text { c: Edad }<45 \text { años } \\
\text { d: Edad }>45 \text { años }\end{array}$ & $\begin{array}{r}\mathrm{pc}=0,474 \\
\mathrm{rdc}=0,4616 \\
\mathrm{rdd}=0,3448\end{array}$ & Sun et al., $2015^{5}$ & \\
\hline $\begin{array}{l}\text { Multifocalidad: } \\
\text { e: Tumor multifocal } \\
\text { f: Tumor unifocal } \\
\end{array}$ & $\begin{aligned} \mathrm{pe} & =0,259 \\
\mathrm{rde} & =0,5358 \\
\mathrm{rdf} & =0,4703\end{aligned}$ & Sun et al., $2015^{5}$ & \\
\hline $\begin{array}{l}\text { Tamaño del tumor: } \\
\mathrm{g}:>2 \mathrm{~cm} \\
\mathrm{~h}:<2 \mathrm{~cm}\end{array}$ & $\begin{array}{l}\mathrm{pg}=0,369 \\
\mathrm{rdg}=0,7212 \\
\mathrm{rdh}=0,4790\end{array}$ & Sun et al., $2015^{5}$ & \\
\hline $\begin{array}{l}\text { Localización del tumor: } \\
\text { i: Área central y polo inferior } \\
\text { j: Polo superior }\end{array}$ & $\begin{array}{r}\mathrm{pi}=0,722 \\
\mathrm{rdi}=0,4672 \\
\mathrm{rdj}=0,3181\end{array}$ & Sun et al., $2015^{5}$ & \\
\hline $\begin{array}{l}\text { Invasión linfovascular: } \\
\text { k: Positiva } \\
\text { I: Negativa }\end{array}$ & $\begin{array}{c}\mathrm{pk}=0,238 \\
\mathrm{rdk}=0,5771 \\
\mathrm{rdl}=0,3378\end{array}$ & Sun et al., $2015^{5}$ & \\
\hline $\begin{array}{l}\text { Extensión extratiroidea: } \\
\text { m: Positiva } \\
\text { n: Negativa }\end{array}$ & $\begin{array}{c}\mathrm{pm}=0,231 \\
\mathrm{rdm}=0,5138 \\
\mathrm{rdn}=0,4852\end{array}$ & Sun et al., $2015^{5}$ & \\
\hline $\begin{array}{l}\text { Invasión Capsular: } \\
\text { o: Positiva } \\
\text { p: Negativa }\end{array}$ & $\begin{array}{l}\mathrm{po}=0,206 \\
\mathrm{rdo}=0,5419 \\
\mathrm{rdp}=0,3714\end{array}$ & Sun et al., $2015^{5}$ & \\
\hline $\begin{array}{l}\text { VPP de la ecografía en } \\
\text { la detección de ganglios positivos }\end{array}$ & VPP $86 \%$ & $\begin{array}{l}\text { Kim et al., } 2008^{13} \\
\text { Zhao y Li }{ }^{14}\end{array}$ & $\begin{array}{l}\text { Con este dato se obtiene } \\
\text { que la proporción de } \\
\text { pacientes cN+ que son pNo. }\end{array}$ \\
\hline $\begin{array}{l}\text { Relación de proporción entre } \\
\text { las poblaciones } \mathrm{cN} 0 \text { y cN+ }\end{array}$ & $\begin{array}{l}\text { - cN+: 9,3\% de la población } \\
\text { - cN0: } 90,7 \% \text { de la población }\end{array}$ & Wada et al., $2003^{6}$ & \\
\hline Proporción de estadios en cN+ con pN+ & $\begin{array}{l}\text { pN1a: } 99 /(99+130)=42 \% \\
\text { pN1b: } 130 /(99+130)=58 \%\end{array}$ & Nixon et al., $2014{ }^{10}$ & $\begin{array}{c}\text { Con este dato se pondera la } \\
\text { supervivencia libre de RLR } \\
\text { global de los pacientes } \mathrm{cN}+ \\
\text { con pN+ }\end{array}$ \\
\hline Proporción de estadios en cN0 con pN+ & $\begin{array}{l}\text { pN1a: } 60 /(60+73)=45 \% \\
\text { pN1b: } 73 /(60+73)=55 \%\end{array}$ & Hartl et al., $2012^{19}$ & \\
\hline Supervivencia libre de RLR a 5 años & $\begin{array}{c}\text { pN0: } 99 \% \\
\text { pN1a: } 93 \% \\
\text { pN1b: } 90 \% \\
\text { Global } \mathrm{cN}+\mathrm{pN}+=0,9126 \\
\text { Global } \mathrm{cN} 0 \mathrm{pN}+=0,9135\end{array}$ & Nixon et al., $2014^{10}$ & \\
\hline Supervivencia libre de RLR a 10 años & $\begin{array}{c}\text { pN0: } 96 \% \\
\text { pN1a: } 88 \% \\
\text { pN1b: } 85 \% \\
\text { Global cN+ pN+ = 0,8626 } \\
\text { Global cN0 pN+ = 0,8635 }\end{array}$ & Nixon et al., $2014^{10}$ & \\
\hline Supervivencia global a 10 años & $\begin{array}{c}\mathrm{N} 0: 98 \% \\
\mathrm{~N} 1 \mathrm{a}: 92 \% \\
\mathrm{~N} 1 \mathrm{~b}: 94 \% \\
\text { Global } \mathrm{cN}+\mathrm{pN}+=0,9316 \\
\text { Global } \mathrm{cN} 0 \mathrm{pN}+=0,9310\end{array}$ & Vrachimis et al., $2015^{20}$ & \\
\hline
\end{tabular}


nidos por los estudios seleccionados. En el anexo 2 se describe la estrategia de simulación genérica que fue usada para cada uno de estos parámetros en el software STATA.

Una vez definida dicha población, se determinó la posibilidad de micrometástasis. Para este paso se utilizó el método de "standard gamble" y se definió un punto de corte en 3 factores de riesgo. Todo paciente con más de tres factores de riesgo presentes se consideraba positivo para micrometástasis. Como prueba de la exactitud del modelo, se determinó la prevalencia de los factores de riesgo y de la presencia de micrometástasis y se comparó con los resultados de la literatura.

Generación de la población $\mathbf{c N + :}$ La población con ganglios clínicamente positivos se generó a partir de los estudios de Kim y col. ${ }^{13}$ y el metaanálisis de Zhao y Li ${ }^{14}$, que reportaron la frecuencia de ganglios sospechosos por ecografía, ajustando por la proporción de falsos positivos (Tabla 1).

Proporción entre las poblaciones cNO y cN+: La distribución de las poblaciones $\mathrm{cN} 0$ y cN+ se obtuvo a partir del estudio de Wada y colaboradores ${ }^{6}$. Finalmente, la población se segmentó en función de sus estadios ganglionares como se muestra en la figura 1.

Simulación de desenlaces: La distribución de los desenlaces (RLR y sobrevivencia) se realizó en función de la variable metástasis ganglionar, usando los porcentajes de riesgo de acuerdo con la estadificación ganglionar reportada en la literatura. Estas distribuciones fueron binomiales. En la figura 2 , se observa un diagrama de proceso de la generación de poblaciones, desenlaces y comparación de los escenarios propuestos.

\section{Análisis estadístico}

Con estas poblaciones generadas se procedió a realizar las comparaciones en escenarios de migración y no migración de estadio ganglionar, para comparar los desenlaces de RLR y supervivencia, entre poblaciones llevadas a vaciamiento ganglionar y poblaciones sin vaciamiento. Se utilizó la prueba de chi cuadrado para variables categóricas y la prueba de t de student para variables continuas. Se considero un valor de $\mathrm{p}<0,05$ como estadísticamente significativo. Para todos las simulaciones y análisis se utilizó el software Stata ${ }^{\circledR}$, versión 14 (StataCorp. LP, College Station, TX, USA).

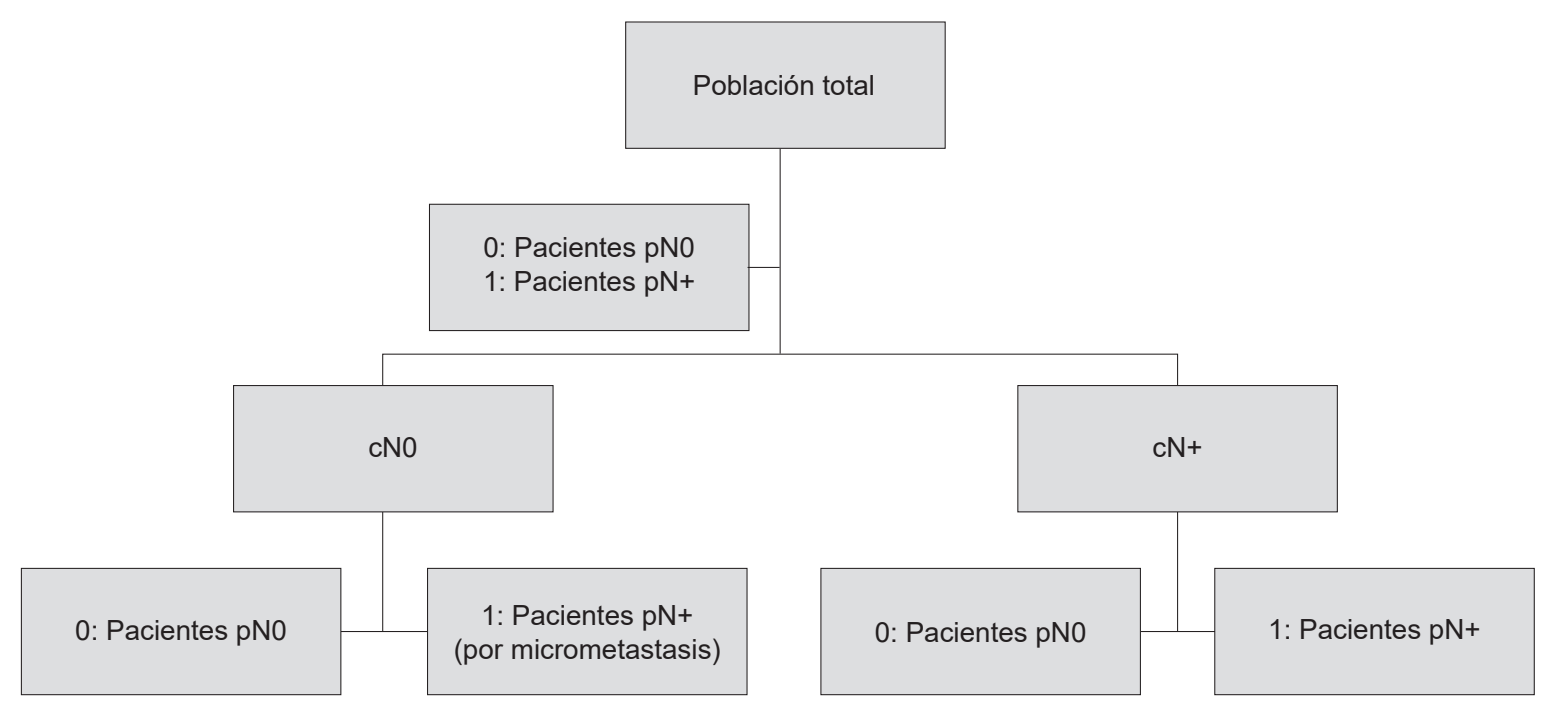

Figura 1. Distribución final de la población hipotética en el escenario de vaciamiento ganglionar. En el escenario de no vaciamiento, la población cNO no se discrimina dado que no se realiza estudio histopatológico ganglionar. 


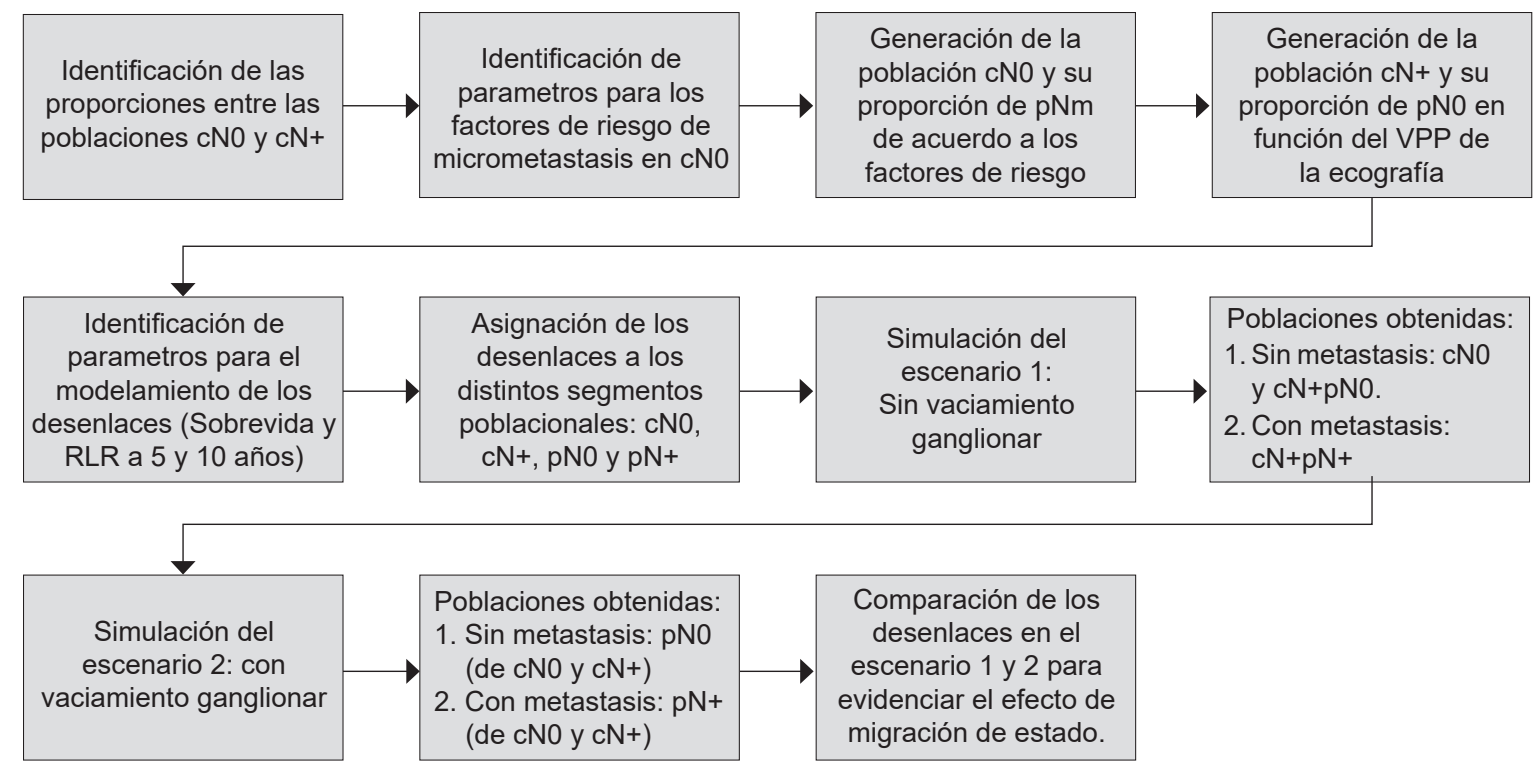

cN0: pacientes sin compromiso ganglionar clínicamente evidente $\mathrm{cN}+$ : pacientes con compromiso ganglionar clínicamente evidente pNm: pacientes con compromiso patológico ganglionar por micrometástasis $\mathrm{pN+:}$ pacientes con compromiso patológico ganglionar metastásico pNO: pacientes sin compromiso patológico ganglionar metastásico

Figura 2. Diagrama de proceso de la generación de poblaciones, desenlaces y comparación de los escenarios propuestos.

\section{Resultados}

En la tabla 1 se describen los parámetros extraídos para el modelo de simulación con su fuente. Se simularon en total 1.000.000 de observaciones.

\section{Desenlace recurrencia loco regional}

Según Ryu y col, ${ }^{15}$, el riesgo de RLR en pacientes cN0 con pN1a fue de 5,7 \% a 6,5 años de seguimiento. Según Hwangbo y col. ${ }^{16}$, el riesgo de recurrencia total a 5 años y 10 años fue de $1,4 \%$ y $2,9 \%$ en pacientes pN0, 6,0 \% y 12,2 \% en pacientes pN1ay $11,4 \%$ y $19,3 \%$ en pacientes pN1b. Nixon y col. ${ }^{17}$ reportaron una supervivencia libre de recurrencia loco regional a los 5 y 10 años así: $99 \%$ y $96 \%$ para pacientes pN0; $93 \%$ y $88 \%$ en pacientes pN1a, y $90 \%$ y $85 \%$ en pacientes pN1b, mientras en otro estudio de Nixon y col. ${ }^{18}$ reportaron pacientes en estadios $\mathrm{pN} 1$ con una supervivencia libre de recurrencia ganglionar central a 5 años de $96,4 \%$, y una tasa de supervivencia libre de recurrencia a 5 años del $91 \%$. Los resultados de
Ryu y col. ${ }^{15}$, Hwangbo y col. ${ }^{16}$ y Nixon y col. ${ }^{17}$ reportaron frecuencias de RLR muy similares en los grupos cN0 y cN+ a 5 años, cuando el estadio patológico es pN1a (5,7 \%, $6 \%$ y $7 \%$ ), por lo que se consideró que la tasa de recurrencia será la misma para el mismo estadio patológico.

La comparación entre los estudios de Wada y col. ${ }^{6}$, Kim y col. ${ }^{13}$, Nixon y col. ${ }^{17}$ y Hartl y col. ${ }^{19}$, demostró que las proporciones de los estadios ganglionares son diferentes entre los pacientes cN0 y cN+. En pacientes cN0 los porcentajes de pN0 se encuentran entre el 40-60\%, mientras que los porcentajes de pN0 en pacientes $\mathrm{cN}+$ es cercano al $14 \%$. De acuerdo con Nixon y col. ${ }^{17}$, la proporción de estadios en pacientes $\mathrm{cN}+$ es cercano al $42 \%$ para estadios pN1a y $58 \%$ para estadios pN1b. Finalmente, según Hartl y col. ${ }^{19}$, la proporción de estadios en pacientes $\mathrm{cN} 0$ con positividad ganglionar al estudio histopatológico, es cercano al $45 \%$ para estadios pN1a y $55 \%$ para estadios pN1b. 
Tabla 2. Características de la población total simulada cN0 (907.300 observaciones).

\begin{tabular}{lccc}
\hline Variables & Valores & Observaciones & Porcentaje \\
\hline \multirow{2}{*}{ Sexo } & 0. Femenino & 772.503 & 85,1 \\
& 1. Masculino & 134.797 & 14,9 \\
Edad & 0. Edad > 45 años & 476.774 & 52,5 \\
& 1. Edad < 45 años & 430.526 & 47,5 \\
Multifocalidad & 0. Tumor unifocal & 671.613 & 74,0 \\
Tamaño del tumor & 1. Tumor multifocal & 235.687 & 26,0 \\
& 0. $<2$ cm & 573.014 & 63,2 \\
Localización del tumor & 1. > 2 cm & 334.286 & 36,8 \\
\multirow{2}{*}{ Invasión linfovascular } & 0. Polo superior & 251.895 & 27,8 \\
& 1. Área central y polo inferior & 655.405 & 72,2 \\
Extensión extratiroidea & 0. Negativa & 690.526 & 76,1 \\
& 1. Positiva & 216.774 & 23,9 \\
Invasión capsular & 0. Negativa & 698.152 & 76,9 \\
& 1. Positiva & 209.148 & 23,1 \\
& 0. Negativa & 719.828 & 79,3 \\
& 1. Positiva & 187.472 & 20,7 \\
\hline
\end{tabular}

\section{Desenlace supervivencia global}

Vrachimis y col. ${ }^{20}$, reportó una supervivencia global a los 10 años según el estadio ganglionar así: $98 \%$ para N0, $92 \%$ para N1a y $94 \%$ para N1b.

\section{Población cN+, cNO a partir de factores de riesgo y población total}

La prevalencia de compromiso micrometastásico fue del $55 \%$, lo cual es compatible con los distintos reportes de la literatura ${ }^{4-6}$. La distribución poblacional fue la siguiente: $9,27 \%$ de los pacientes son $\mathrm{cN}+\mathrm{y} 90,73 \%$ de los pacientes son $\mathrm{cN} 0$.

En la tabla 2 se observan las características de la población total $\mathrm{CN} 0$ simulada de acuerdo con sus factores de riesgo. El número de observaciones de la población cN0 fue de 907.300 y el de la población $\mathrm{cN}+$ fue de 93.700 , para un universo total de población de 1.000.000 de observaciones. El compromiso ganglionar de ambas poblaciones (cN0 y cN+) puede observarse en la tabla 3.

\section{Análisis de la migración}

El escenario inicial en el cual no se realiza vaciamiento central profiláctico a ningún paciente, se muestra en la tabla 4. En el escenario donde se realiza vaciamiento ganglionar profiláctico y se
Tabla 3. Segmentos poblacionales y sus estadios ganglionares.

\begin{tabular}{cccc}
\hline $\begin{array}{c}\text { Segmento } \\
\text { poblacional }\end{array}$ & $\begin{array}{c}\text { Compromiso } \\
\text { ganglionar }\end{array}$ & Observaciones & Porcentaje \\
\hline \multirow{2}{*}{ cN0 } & 0. pN0 & 415.871 & 45,8 \\
& 1. pN1 & 491.429 & 54,2 \\
\multirow{2}{*}{ cN+ } & 0. pN0 & 12.876 & 13,9 \\
& 1. pN1 & 79.824 & 86,1 \\
Totales & & 1.000 .000 & 100 \\
\hline
\end{tabular}

redistribuye la población de acuerdo a su estadio ganglionar, los pacientes con positividad ganglionar (micro y macrometástasis) son asignados al grupo de $\mathrm{cN}+$. Se observa entonces la paradoja epidemiológica de Will Rogers, con un aumento aparente de la supervivencia libre de RLR y de la supervivencia general a 10 años en el grupo cN0, mientras los desenlaces del segmento poblacional cN+ varían de manera mínima y en la población general permanecen sin cambios, al tiempo que el conteo de individuos que sufren un desenlace determinado permanece sin cambio alguno. Lo anterior se evidencia claramente en la tabla 5. La figura 3 demuestra la distribución poblacional en ambos escenarios. 
Tabla 4. Desenlaces para ambos segmentos poblacionales en el escenario de no realizar vaciamiento ganglionar profiláctico.

\begin{tabular}{lccccc}
\hline $\begin{array}{c}\text { Segmento } \\
\text { poblacional }\end{array}$ & $\begin{array}{c}\text { Compromiso } \\
\text { ganglionar }\end{array}$ & $\begin{array}{c}\text { Supervivencia libre } \\
\text { de RLR a 5 años }\end{array}$ & $\begin{array}{c}\text { Supervivencia libre } \\
\text { de RLR a 10 años }\end{array}$ & $\begin{array}{c}\text { Supervivencia global } \\
\text { a 10 años }\end{array}$ & $\begin{array}{c}\text { Población } \\
\text { total }\end{array}$ \\
\hline cN0 & NA & 860.521 & 824.071 & 864.906 & 907.300 \\
& & $(94,8 \%)$ & $(90,8 \%)$ & $(95,3 \%)$ & 12.834 \\
& 0. pN0 & 12.766 & 12.367 & $(98,1 \%)$ & 79.824 \\
cN+ & & $(99,1 \%)$ & $(96,1 \%)$ & 74.364 & \\
& 1. pN+ & 72.767 & 68.731 & $(93,2 \%)$ & \\
Total: & & $(91,2 \%)$ & $(86,1 \%)$ & 877.540 & 920.176 \\
cN0 y cN+pN0 & & 873.287 & 836.438 & $(95,4 \%)$ & \\
Población total & & $(94,9 \%)$ & $(90,9 \%)$ & $951 ., 904$ & 1.000 .000 \\
\hline
\end{tabular}

Nota: el segmento poblacional $\mathrm{cN}+$ que no demostró compromiso metastásico en la patología se reasigna al segmento cN0. No hay datos de compromiso ganglionar en el grupo $\mathrm{cNO}$, puesto que no se realizó vaciamiento ganglionar profiláctico en este escenario de simulación.
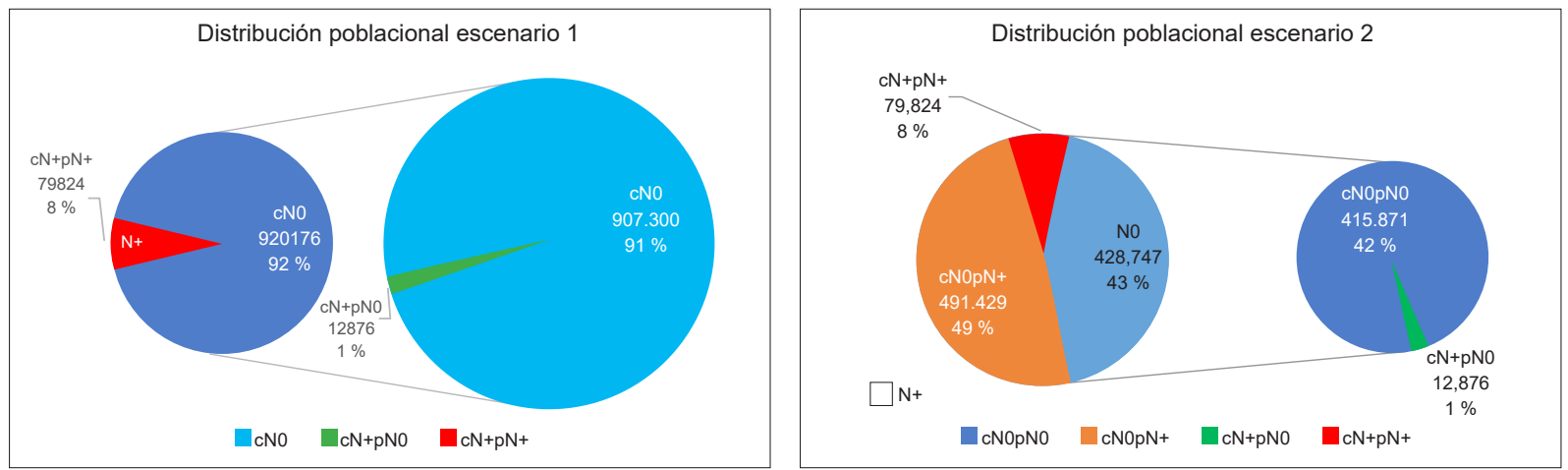

Figura 3. Poblaciones simuladas en el escenario de no vaciamiento (escenario 1) y vaciamiento ganglionar profiláctico (escenario 2), respectivamente.

Tabla 5. Desenlaces para ambos segmentos poblacionales con vaciamiento ganglionar profiláctico y migración de estadio de los pN1 del grupo cNO al grupo cN+.

\begin{tabular}{|c|c|c|c|c|c|}
\hline $\begin{array}{l}\text { Segmento } \\
\text { poblacional }\end{array}$ & $\begin{array}{c}\text { Compromiso } \\
\text { ganglionar }\end{array}$ & $\begin{array}{c}\text { Supervivencia } \\
\text { libre de RLR a } \\
5 \text { años }\end{array}$ & $\begin{array}{c}\text { Supervivencia } \\
\text { libre de RLR a } \\
10 \text { años }\end{array}$ & $\begin{array}{l}\text { Supervivencia } \\
\text { global a } \\
10 \text { años }\end{array}$ & $\begin{array}{c}\text { Total de } \\
\text { observaciones }\end{array}$ \\
\hline \multirow{2}{*}{$\mathrm{cNO}$} & 0. pNO & $\begin{array}{l}411.771 \\
(99,0 \%)\end{array}$ & $\begin{array}{l}399.301 \\
(96,0 \%)\end{array}$ & $\begin{array}{c}407 ., 490 \\
(98,0 \%)\end{array}$ & 415.871 \\
\hline & 1. $\mathrm{pN}+$ & $\begin{array}{l}448.750 \\
(91,3 \%)\end{array}$ & $\begin{array}{l}424.770 \\
(86,4 \%)\end{array}$ & $\begin{array}{l}457.416 \\
(93,1 \%)\end{array}$ & 491.429 \\
\hline \multirow{2}{*}{$\mathrm{cN}+$} & 0. pNO & $\begin{array}{c}12.766 \\
(99,1 \%)\end{array}$ & $\begin{array}{c}12.367 \\
(96,1 \%)\end{array}$ & $\begin{array}{c}12.634 \\
(98,1 \%)\end{array}$ & 12.876 \\
\hline & 1. $\mathrm{pN}+$ & $\begin{array}{c}72.767 \\
(91,2 \%)\end{array}$ & $\begin{array}{c}68.731 \\
(86,1 \%)\end{array}$ & $\begin{array}{c}74.364 \\
(93,2 \%)\end{array}$ & 79.824 \\
\hline Total pN0 & & $\begin{array}{l}424.537 \\
(99,0 \%)\end{array}$ & $\begin{array}{l}411.668 \\
(96,0 \%)\end{array}$ & $\begin{array}{l}420.124 \\
(98,0 \%)\end{array}$ & 428.747 \\
\hline Total pN+ & & $\begin{array}{l}521.517 \\
(91,3 \%)\end{array}$ & $\begin{array}{l}493.501 \\
(86,4 \%)\end{array}$ & $\begin{array}{l}531.780 \\
(93,1 \%)\end{array}$ & 571,253 \\
\hline Totales & & $\begin{array}{l}946.054 \\
(94,6 \%)\end{array}$ & $\begin{array}{l}905.169 \\
(90,5 \%)\end{array}$ & $\begin{array}{l}951.904 \\
(95,2 \%)\end{array}$ & 1.000 .000 \\
\hline
\end{tabular}




\section{Discusión}

El fenómeno de Will Rogers es reconocido como uno de los sesgos más importantes que limitan el uso de grupos históricos de control en ensayos de tratamiento experimental. Esto debido a que el uso de diferentes criterios o herramientas diagnósticas puede generar falsas mejoras en los desenlaces de los grupos de pacientes, los cuales pueden ser interpretados erróneamente como efectos de tratamiento ${ }^{7,10}$.

La observación de un incremento en la proporción de pacientes con positividad ganglionar sobre el tiempo es conocido como migración de estadio ganglionar. Si este fenómeno es acompañado por una distribución de estadios $\mathrm{T}$ constante, existe el potencial de una mejoría paradójica en la supervivencia de los grupos con ganglios positivos y negativos, sin un incremento en la supervivencia global o en los pacientes individuales ${ }^{11}$.

Esta paradoja contrasta con la percepción, generalmente aceptada, de que las nuevas tecnologías de estadiaje generalmente resultan en una detección más temprana y una mejoría de los tratamientos, por ejemplo, de las metástasis ganglionares, llevando a una mejoría de la supervivencia general. Esta forma de reclasificación es bien conocida en la literatura oncológica, y generalmente ocurre después de la introducción de nuevos métodos de imagen, pero nunca ha sido evaluada para el vaciamiento ganglionar profiláctico en cáncer papilar de tiroides, el cual puede jugar un rol al reclasificar los pacientes, haciendo evidente la positividad ganglionar antes ignorada.

En este artículo se ha simulado una población de pacientes con cáncer papilar de tiroides en función de sus factores de riesgo. Según lo publicado previamente, se han definido unas prevalencias de compromiso micrometastásico para los pacientes cN0, así como se ha definido una población de pacientes $\mathrm{cN}+$. De acuerdo con dichos datos, pudo asignarse también una distribución de estadios ganglionares, en donde se evidenció que las relaciones entre los estadios pN1a y pN1b eran similares, tanto para el segmento poblacional cN0 como para los pacientes $\mathrm{cN}+$, de 45:55 y 42:58, respectivamente ${ }^{17,19}$. En cambio, la gran diferen- cia en la distribución de estadio ganglionar se encuentra en la proporción de pacientes pN0 en las poblaciones cN0 y cN+, que correspondía al 45,8 y $13,8 \%$, respectivamente (tabla 3 ).

Resulta interesante notar que, a la luz de la literatura, no existe evidencia suficiente para afirmar que los desenlaces de los pacientes con compromiso ganglionar micrometastásico ( $\mathrm{pNm})$, sean diferentes de los pacientes con compromiso ganglionar clínicamente evidente $(\mathrm{cN}+)$, en términos de supervivencia libre de recurrencia loco regional ${ }^{15,16,17}$.

Cuando se simulan ambos escenarios, llevando la población a vaciamiento ganglionar profiláctico, lo cual implica su re-estadificación, o sin realizarlo, se observa el impacto de la migración del estadio ganglionar sobre los desenlaces de supervivencia libre de RLR y supervivencia global.

En el primer escenario, sin vaciamiento ganglionar profiláctico, la supervivencia libre de RLR a 5 años y a 10 años y la supervivencia global a 10 años, del grupo NO (que incluye los cN0 y los pacientes $\mathrm{cN}+$ pero sin metástasis en el estudio patológico: pN0), son de 94,9\%, 90,9\% y 95,4\%, respectivamente, mientras estos mismos desenlaces para el segmento poblacional $\mathrm{cN}+$ son de $91,1 \%, 86,1 \%$ y $93,2 \%$, respectivamente. Las diferencias entre todos los desenlaces de ambos grupos alcanzan la significancia estadística al comparar las proporciones con la prueba de chi cuadrado, con un valor $\mathrm{p}<0,05$.

En el segundo escenario, usando la misma población y adicionando el vaciamiento ganglionar profiláctico y la consecuente re-estadificación, observamos que todo el grupo $\mathrm{cN} 0 \mathrm{pN}+$ pasa al grupo de $\mathrm{cN}+$, configurando así una supervivencia libre de RLR a 5 años y a 10 años y una supervivencia global a 10 años, del grupo pN0 de 99,0\%, $96,0 \%$ y $98,0 \%$, respectivamente, mientras estos mismos desenlaces para el segmento poblacional $\mathrm{pN}+$, en el cual se incluye ahora el grupo cN0 pN+, es de $91,6 \%, 86,6 \%$ y 93,2 \%, respectivamente. Las diferencias entre todos los desenlaces de ambos grupos alcanzan la significancia estadística al comparar las proporciones con la prueba de chi cuadrado, con un valor $\mathrm{p}<0,05$. 
Cuando se realizan los cálculos de supervivencia libre de RLR a 5 años y a 10 años y de supervivencia global a 10 años, para la población total en el segundo escenario, no hay variaciones en absoluto, y los conteos de observaciones tampoco varían para ninguno de los desenlaces, tal como se evidencia en la tabla 5.

Con este segundo escenario, se hace evidente una mejoría en los desenlaces del grupo N0 (pN0 en el segundo escenario) después del vaciamiento y la re-estadificación de la población, de 94,9\%, $90,9 \%$ y $95,4 \%$ a 99,0 \%, 96,0 \% y 98,0\% para la supervivencia libre de RLR a 5 años y a 10 años y la supervivencia global a 10 años, estas diferencias también son significativas al comparar las proporciones con la prueba de chi cuadrado, con un valor $\mathrm{p}<0,05$.

Al comparar la disminución del riesgo en ambos grupos de pacientes $\mathrm{N} 0$ en los escenarios descritos, se encuentra una disminución absoluta del riesgo de RLR a 5 años y a 10 años de 4,1 y 5,2\%, respectivamente, el cual es un valor similar al reportado en la literatura de 3,9 y $6,9 \%{ }^{21,22}$.

Además, la incidencia de RLR en pacientes cN0 a los 5 años es cercana al $1 \%$, lo cual se corrobora en otros estudios retrospectivos de vaciamiento ganglionar profiláctico en pacientes $\mathrm{cN} 0{ }^{23}$.

$\mathrm{Al}$ analizar el grupo $\mathrm{N}+$ en ambos escenarios, se encuentra que los cambios son mínimos, de $91,2 \%, 86,1 \%$ y $93,2 \%$ a $91,6 \%, 86,6 \%$ y $93,2 \%$, respectivamente, para cada uno de los desenlaces evaluados, y al realizar la prueba de significancia estadística de chi cuadrado, con un valor $\mathrm{p}<0,05$, solo hay mejoría estadísticamente significativa en el desenlace de supervivencia libre de recurrencia locorregional a 10 años. Por su parte, la supervivencia libre de recurrencia locorregional a 5 años y la supervivencia total no presentan cambios estadísticamente significativos.

Por último, al revisar el número de observaciones en cada escenario y los porcentajes totales de la población completa en ambos escenarios, estos no cambian en absoluto y se mantienen en 946.542 (94,6 \%) sujetos sin RLR a 5 años, 904.933 (90,5 \%) sujetos sin RLR a 10 años y 951.904 (95,2 \%) sujetos vivos a los 10 años.
Los hallazgos aquí presentados, a partir de los métodos de simulación usados para representar las poblaciones descritas de pacientes con cáncer papilar de tiroides, han sido observados desde 1985, cuando Alvan Feinstein, propuso el nombre de "fenómeno Will Rogers" para describir la migración de estadio que se observa en pacientes con cáncer de pulmón. En su trabajo original describió los cambios observados en las tasas aparentes de supervivencia de los grupos de estadificación de los pacientes con cáncer de pulmón debido a la mejoría en la sensibilidad de las herramientas diagnósticas, que permitía evidenciar compromiso tumoral ganglionar antes no evidenciado, y por tanto, se presentaba una migración de pacientes entre distintos grupos de estadificación. En este estudio se demostró cómo el incremento en la proporción de pacientes con positividad ganglionar en el tiempo, acompañado de una distribución de estadios $\mathrm{T}$ constante, produce una aparente pero falsa mejoría en el pronóstico específico de cada grupo de estadificación, mientras el pronóstico individual y total de la población de pacientes no cambia $^{12}$.

Después de los hallazgos de Feinstein, múltiples estudios en oncología han demostrado que las nuevas herramientas de imagen permiten la detección de metástasis cancerosas antes de que éstas sean clínicamente evidentes, como consecuencia, más pacientes serán clasificados en estadios más avanzados de enfermedad metastásica a partir de un estadio de menor gravedad, tal migración de estadio resulta en una mejoría aparente de la supervivencia de los pacientes en ambos grupos de estadificación y ha sido demostrado en múltiples patologías, como cáncer de pulmón, próstata, mama y ano ${ }^{8-12}$. Inclusive, dicha paradoja epidemiológica se ha demostrado en escenarios no oncológicos, como la esclerosis múltiple ${ }^{7}$.

De esta manera, usando herramientas y modelos de simulación, logramos un acercamiento a la determinación del impacto del fenómeno de migración de estadio ganglionar en la supervivencia de pacientes con cáncer papilar de tiroides llevados a vaciamiento ganglionar profiláctico, y cómo la mejoría de los desenlaces documentada 
en varios metaanálisis puede ser atribuida a un fenómeno de migración de estadio.

\section{Consideraciones éticas}

Consentimiento informado: Este estudio es una revisión de la literatura, y como tal, no hay necesidad de un consentimiento informado. El Comité de Ética Institucional aprobó el diseño y la metodología del estudio.

Declaración de conflicto de intereses: Los autores declaran no tener ningún conflicto de intereses.

Fuentes de financiación: trabajo financiado con recursos de los autores.

\section{Contribución de los autores:}

- Concepción y diseño del estudio: José Eduardo AgamezFuentes, Álvaro Enrique Sanabria.

- Adquisición de datos: José Eduardo Agamez-Fuentes, Álvaro Enrique Sanabria.

- Análisis e interpretación de datos: José Eduardo Agamez-Fuentes, Álvaro Enrique Sanabria.

- Redacción del manuscrito: José Eduardo AgamezFuentes, Álvaro Enrique Sanabria.

- Revisión crítica: José Eduardo Agamez-Fuentes, Álvaro Enrique Sanabria.

\section{Referencias}

1. Carling T, Carty SE, Ciarleglio MM, Cooper DS, Doherty GM, Kim LT, et al. American Thyroid Association design and feasibility of a prospective randomized controlled trial of prophylactic central lymph node dissection for papillary thyroid carcinoma. Thyroid. 2012;22:237-44. https://doi.org/10.1089/thy.2011.0317

2. Chen L, Wu YH, Lee CH, Chen HA, Loh EW, Tam KW. Prophylactic central neck dissection for papillary thyroid carcinoma with clinically uninvolved central neck lymph nodes: A systematic review and meta-analysis. World J Surg. 2018;42:2846-57. https://doi.org/10.1007/s00268-018-4547-4

3. Zhao WJ, Luo H, Zhou YM, Dai WY, Zhu JQ. Evaluating the effectiveness of prophylactic central neck dissection with total thyroidectomy for cN0 papillary thyroid carcinoma: An updated meta-analysis. Eur J Surg Oncol. 2017;43:1989-2000. https://doi.org/10.1016/j.ejso.2017.07.008

4. Chunhao L, Liu Y, Zhang L, Dong Y, Hu S, Xia Y, et al. Risk factors for high-volume lymph node metastases in $\mathrm{cN} 0$ papillary thyroid microcarcinoma. Gland Surg. 2019;8:550-6.

https://doi.org/10.21037/gs.2019.10.04
5. Sun W, Lan X, Zhang H, Dong W, Wang Z, He L, et al. Risk factors for central lymph node metastasis in CNO papillary thyroid carcinoma: A systematic review and meta-analysis. Plos One. 2015;10: e0139021. https://doi.org/10.1371/journal.pone.0139021

6. Wada N, Duh QY, Sugino K, Iwasaki H, Kameyama K, Mimura T, et al. Lymph node metastasis from 259 papillary thyroid microcarcinomas: frequency, pattern of occurrence and recurrence, and optimal strategy for neck dissection. Ann Surg. 2003;237:399-407.

https://doi.org/10.1097/01.SLA.0000055273.58908.19

7. Sormani MP. The Will Rogers phenomenon: the effect of different diagnostic criteria. J Neurol Sci. 2009;287 Suppl 1:S46-49.

https://doi.org/10.1016/S0022-510X(09)71300-0

8. Chee KG, Nguyen DV, Brown M, Gandara DR, Wun T, Lara PN. Positron emission tomography and improved survival in patients with lung cancer: The Will Rogers phenomenon revisited. Arch Intern Med. 2008;168:1541-9. https://doi.org/10.1001/archinte.168.14.1541

9. Gofrit ON, Zorn KC, Steinberg GD, Zagaja GP, Shalhav AL. The Will Rogers phenomenon in urological oncology. J Urol. 2008;179:28-33. https://doi.org/10.1016/j.juro.2007.08.125

10. Tan GH, Bhoo-Pathy N, Taib NA, See MH, Jamaris S, Yip CH. The Will Rogers phenomenon in the staging of breast cancer - does it matter? Cancer Epidemiol. 2015;39:115-7. https://doi.org/10.1016/j.canep.2014.11.005

11. Sekhar H, Zwahlen M, Trelle S, Malcomson L, Kochhar R, Saunders MP, et al. Nodal stage migration and prognosis in anal cancer: A systematic review, meta-regression, and simulation study. Lancet Oncol. 2017;18:1348-59. https://doi.org/10.1016/S1470-2045(17)30456-4

12. Feinstein AR, Sosin DM, Wells CK. The Will Rogers phenomenon. Stage migration and new diagnostic techniques as a source of misleading statistics for survival in cancer. N Engl J Med. 1985;312:1604-8. https://doi.org/10.1056/NEJM198506203122504

13. Kim E, Park JS, Son KR, Kim JH, Jeon SJ, Na DG. Preoperative diagnosis of cervical metastatic lymph nodes in papillary thyroid carcinoma: Comparison of ultrasound, computed tomography, and combined ultrasound with computed tomography. Thyroid. 2008;18:411-8. https://doi.org/10.1089/thy.2007.0269

14. Zhao H, Li H. Meta-analysis of ultrasound for cervical lymph nodes in papillary thyroid cancer: Diagnosis of central and lateral compartment nodal metastases. Eur J Radiol. 2019;112:14-21. https://doi.org/10.1016/j.ejrad.2019.01.006

15. Ryu YJ, Cho JS, Park MH, Yoon JH. Identifying risk factors of recurrence for clinically node negative papillary thyroid carcinoma with pathologic N1a. BMC Surgery. 2019;19:78. https://doi.org/10.1186/s12893-019-0541-5 
16. Hwangbo Y, Kim JM, Park YJ, Lee EK, Lee YJ, Park DJ, et. al. Long-term recurrence of small papillary thyroid cancer and its risk factors in a korean multicenter study. J Clin Endocrinol Metab. 2017;102:625-33. https://doi.org/10.1210/jc.2016-2287

17. Nixon IJ, Wang LY, Palmer FL, Tuttle RM, Shaha AR, Shah JP, et al. The impact of nodal status on outcome in older patients with papillary thyroid cancer. Surgery. 2014;156:137-46. https://doi.org/10.1016/j.surg.2014.03.027

18. Nixon IJ, Wang LY, Ganly I, Patel SG, Morris LG, Migliacci JC, et al. Outcomes for patients with papillary thyroid cancer who do not undergo prophylactic central neck dissection. Br J Surg. 2016;103:218-25. https://doi.org/10.1002/bjs.10036

19. Hartl DM, Leboulleux S, Ghuzlan AA, Baudin E, Chami L, Schlumberger M, Travagli JP. Optimization of staging of the neck with prophylactic central and lateral neck dissection for papillary thyroid carcinoma. Ann Surg. 2012;255:777-83.

https://doi.org/10.1097/SLA.0b013e31824b7b68
20. Vrachimis A, Wenning Ch, Gerß J, Dralle H, Tabassi MV, Schober O, Riemann B, MSDS study group. Not all DTC patients with $\mathrm{N}$ positive disease deserve the attribution "high risk". Contribution of the MSDS Trial. J Surg Oncol. 2015;112:9-14. https://doi.org/10.1002/jso.23948

21. Lang BH, Ng SH, Lau LL, Cowling BJ, Wong KP, Wan KY. A systematic review and meta-analysis of prophylactic central neck dissection on short-term locoregional recurrence in papillary thyroid carcinoma after total thyroidectomy. Thyroid. 2013;23:1087-98.

https://doi.org/10.1089/thy.2012.0608

22. Barczyński M, Konturek A, Stopa M, Nowak W. Prophylactic central neck dissection for papillary thyroid cancer. Br J Surg. 2013;100:410-8.

https://doi.org/10.1002/bjs.8985

23. Xue S, Wang P, Liu J, Li R, Zhang L, Chen G. Prophylactic central lymph node dissection in cN0 patients with papillary thyroid carcinoma: A retrospective study in China. Asian J Surg. 2016;39:131-6.

https://doi.org/10.1016/j.asjsur.2015.03.015

\section{Anexo 1. Estrategia de búsqueda en PubMed}

"thyroid"[All Fields] AND ("cancer"[All Fields] OR "carcinoma"[All Fields]) AND "papillary"[All Fields] AND "prophylactic"[All Fields] AND central[All Fields] AND "neck"[All Fields] AND "dissection"[All Fields] AND ("2010/04/18"[PDat]: “2020/04/14"[PDat])

\section{Anexo 2. Salida de las simulaciones para población cNO y micrometástasis}

clear

** Simularemos una población de 1000000 de pacientes:

set obs 100000

** Fijamos la semilla:

set seed 2345

** Riesgo de muerte o desenlace que se desee estudiar del estado dicotomico "b" de la variable estudiada: por ejemplo aca rdb puede ser el riesgo de muerte de los pacientes con nodulos negativos: scalar $\mathrm{rdb}=0.15$

** Riesgo de muerte o desenlace que se desee estudiar del estado dicotomico "a", la cual es la complementaria en el desenlace dicotomico " $b$ ", por ejemplo aca seria el riesgo de muerte de los pacientes con nodulos positivos:

scalar rda $=0.65$

** Ahora definimos la prevalencia del estado "a", por ejemplo a continuacion se define una prevalencia del $25 \%$ de nodulos positivos:

scalar pa $=0.25$

** Ahora definimos el $\mathrm{n}=1$ para la distribución binomial, convirtiendola así en un bernoulli:

generate $\mathrm{n}=1$

** A continuacion definimos la funcion de masa de probabilidad (distribución de bernoulli) del estado "a" de la variable a estudiar, por ejemplo compromiso ganglionar:

generate $a=r b i n o m i a l(n, p a)$

** Tabulamos la funcion masa de probabilidad para ver los eventos:

tab a

** Generamos la función masa de probabilidad del desenlace a estudiar para la población de acuerdo los estados de la variable, en este caso muertes generate $\mathrm{m}=(1-\mathrm{a}) *$ rbinomial $(\mathrm{n}, \mathrm{rdb})+\mathrm{a}^{*}$ rbinomial(n,rda) 